\title{
Trends and Complications of Arthroscopic-Assisted Tibial Plateau Fracture Fixation: A Matched Cohort Analysis
}

\author{
Alan G. Shamrock, M.D., Zain Khazi, B.S., Trevor R. Gulbrandsen, M.D., \\ Kyle R. Duchman, M.D., Michael C. Willey, M.D., Matthew D. Karam, M.D., \\ Matthew H. Hogue, M.D., and J. Lawrence Marsh, M.D.
}

\begin{abstract}
Purpose: To determine trends in arthroscopic-assisted tibial plateau fracture fixation (AATPFF), to evaluate trends in the overall rate of tibial plateau fracture fixation, and to compare postoperative complications between AATPFF and traditional tibial plateau fixation. Methods: A retrospective review of patients undergoing AATPFF and traditional tibial plateau fixation was conducted using the Humana Inc. administrative database from 2007 to 2016. A 1:1 propensity match was utilized to match patients in the 2 study groups based on age, sex, obesity, diabetes, hypertension, chronic obstructive pulmonary disease, depression or anxiety, and smoking history. Postoperative complications were grouped as minor medical complications, major medical complications, surgical complications, emergency department visits, and reoperation. Linear regression analysis was used to assess trends and Pearson's $\chi^{2}$ test was used to compare postoperative complications with statistical significance defined as $P<.05$. Results: In total, 522 patients underwent AATPFF and 3920 patients underwent traditional tibial plateau fracture fixation. There was a 4-fold increase in the use of AATPFF over the study period $(P=.0173)$. Similarly, there was an increase in the utilization of traditional tibial plateau fracture fixation, although to a lesser extent (1.33-fold). After propensity matching, the traditional fixation group demonstrated significantly higher rates of minor medical complications $(8.2 \%$ vs $2.7 \%, P=.0002)$, major medical complications $(9.9 \%$ vs $4.6 \%, P=.0018)$, surgical complications ( $13.2 \%$ vs $2.7 \%, P<.0001$ ), and emergency department visits $(21.4 \%$ vs $13.5 \%$, $P<.0001)$ within 90 days of surgery compared with the AATPFF group. There was no difference in reoperation rates within 90 days between the 2 groups $(2.9 \%$ vs $3.6 \%, P=.85)$. Conclusions: The incidence of tibial plateau fracture fixation is increasing, however, use of AATPFF is increasing at a faster rate compared to traditional techniques. Furthermore, the addition of knee arthroscopy to fracture fixation does not increase the risk of complication, reoperation, or emergency department visit within 90 days. Level of Evidence: III, retrospective matched cohort.
\end{abstract}

$\mathbf{T}$ ibial plateau fractures are estimated to represent approximately $1 \%$ of all fractures and typically occur in older patients with osteoporotic bone or young patients involved in high-energy trauma. ${ }^{1-3}$ Although

From the Department of Orthopaedic Surgery, University of Iowa Hospitals and Clinics, Iowa City, Iowa, U.S.A.

The authors report that they have no conflicts of interest in the authorship and publication of this article. Full ICMJE author disclosure forms are available for this article online, as supplementary material.

Received January 13, 2020; accepted June 30, 2020.

Address correspondence to Alan Shamrock, M.D., Department of Orthopaedic Surgery, 200 Hawkins Dr., Iowa City, IA 52242. E-mail: Alan-shamrock@uiowa.edu

(C) 2020 THE AUTHORS. Published by Elsevier Inc. on behalf of the Arthroscopy Association of North America. This is an open access article under the CC BY-NC-ND license (http://creativecommons.org/licenses/by-nc-nd/4.0/). 2666-061X/2047

https://doi.org/10.1016/j.asmr.2020.06.016 uncommon, tibial plateau fractures comprise a broad spectrum of fracture morphologies with differing degrees of articular injury. The current standard of care is open reduction and internal fixation with the goal of achieving anatomic joint reduction, recreating normal limb alignment, and restoring joint stability to promote early mobilization and knee range of motion. ${ }^{4}$ Given the severity of subchondral bone injury, the reduction of tibial plateau fractures often poses a significant challenge. Traditional techniques involve large open approaches with articular reduction assessed directly by visualizing the joint using an arthrotomy or indirectly using fluoroscopy. Krause et al. ${ }^{5}$ evaluated the joint surface of tibial plateau fractures managed with traditional fixation techniques using arthroscopy and found that only $41.2 \%$ had a satisfactory reduction.

Knee arthroscopy is a minimally invasive technique that allows direct visualization of the articular surface 
during fracture reduction. ${ }^{4,6}$ Arthroscopic-assisted tibial plateau fracture fixation (AATPFF) was described by Jennings $^{7}$ and Caspari et al. $^{8}$ in 1985. Potential advantages of AATPFF include direct confirmation of adequate articular reduction, less soft-tissue dissection, avoidance of an arthrotomy that places the anterior horn of the lateral meniscus at risk, and the diagnosis and treatment of concomitant intra-articular meniscal and cruciate ligament pathology, which is estimated to occur in up to $71 \%{ }^{4}$ of tibial plateau fractures. ${ }^{9}$ Multiple studies have demonstrated good-to-excellent radiographic and clinical outcomes at short-term follow-up, particularly in the setting of isolated lateral (Schatzker I-III) fracture patterns. ${ }^{10-16}$

Currently, the complication rate of AATPFF is poorly understood. In addition, arthroscopy is not frequently performed by orthopaedic traumatologists, and the rate of use of concomitant knee arthroscopy in the treatment of tibial plateau fractures is unknown. The purposes of the current study were to determine trends in AATPFF, to evaluate trends in the overall rate of tibial plateau fracture fixation, and to compare postoperative complications between AATPFF and traditional tibial plateau fixation techniques.

\section{Methods}

The current study was deemed to be exempt from institutional review board approval.

\section{Database}

The PearlDiver research tool (PearlDiver Technologies, Colorado Springs, CO) is a research source that queries available patients in the Humana administrative claims database (Humana, Louisville, JY). This customizable tool can access deidentified information from more than 20 million patients who are insured privately or through Medicare. Patient information, including demographic characteristics, operative procedures performed, comorbidities, and complications, can be obtained using International Classification of Diseases Ninth Revision and Tenth Revision codes; Current Procedural Terminology (CPT) codes; and National Drug Codes. This system provides access to a sizeable cohort of patients across various hospital settings. In addition, these patients can be longitudinally tracked over time, allowing for analysis of postoperative complications.

\section{Patient Selection}

Patients undergoing AATPFF and open tibial plateau fracture fixation from the years 2007 to 2016 were identified in the Humana database (HOrtho) using CPT codes. The following CPT codes were used to identify the traditional open tibial plateau group: 27535 and 27536 (open treatment of tibial fracture, proximal [plateau]). CPT codes 29855 and 29856 (arthroscopically aided treatment of tibial fracture, proximal [plateau]) were used to identify the AATPFF group.

The annual incidence of open and arthroscopic-assisted tibial plateau fracture procedures was recorded. Patient demographics, comorbidities, and 90-day postoperative complications were queried using relevant International Classification of Diseases codes. Demographic characteristics and medical comorbidities analyzed included age, sex, obesity, diabetes, hypertension, chronic obstructive pulmonary disease, Ehlers-Danlos syndrome, and smoking history. In addition, psychiatric conditions, including major depressive disorder, generalized anxiety disorder, and fibromyalgia, were included. The following reported 90-day postoperative complications were included in this study: postoperative pneumonia, urinary tract infection, blood transfusion, sepsis, septic shock, venous thromboembolism, myocardial infarction, superficial surgical-site infection, deep surgical-site infection, wound dehiscence, postoperative peripheral nerve palsy, emergency department visits, and associated reoperations.

\section{Statistical Analysis}

A 1:1 propensity match was used in an effort to minimize confounding by matching patients in the 2 study groups based on age, sex, obesity, diabetes, hypertension, chronic obstructive pulmonary disease, major depressive disorder or generalized anxiety disorder, and smoking history. Postoperative complications were grouped as minor medical complications (pneumonia, urinary tract infection, and blood transfusion), major medical complications (sepsis, septic shock, venous thromboembolism, and myocardial infarction), surgical complications (superficial surgical site infection, deep surgical-site infection, wound dehiscence, and postoperative peripheral nerve palsy), emergency department visits, and reoperation. Linear regression analysis was used to assess trends and Pearson's $\chi^{2}$ test was used to compare postoperative complications with significance defined as $P<.05$. All statistical analyses were performed using the open-source R software (R Foundation for Statistical Computing, Vienna, Austria) contained within the PearlDiver research tool.

\section{Results}

\section{Temporal Trends for Tibial Plateau Fracture Fixation}

During the study period, a total of 522 patients and 3920 patients underwent AATPFF and traditional tibial plateau fixation, respectively. There was a significant increase AATPFF use over time $(P=.0173)$ with a 4-fold increase observed from 2007 to 2016 (1.1 per 100,000 persons in 2007 to 4.1 per 100,000 persons in 2017; Fig 1). Similarly, there was an increase in the use of traditional tibial plateau fracture fixation, although at a much slower rate than AATPFF (1.33-fold increase from 2007 to 2016; Fig 1). 


\section{Trends in Surgical Managment of Tibial Plateau Fractures}

Fig 1. Trends in the management of tibial plateau fractures from 2007 to 2016.

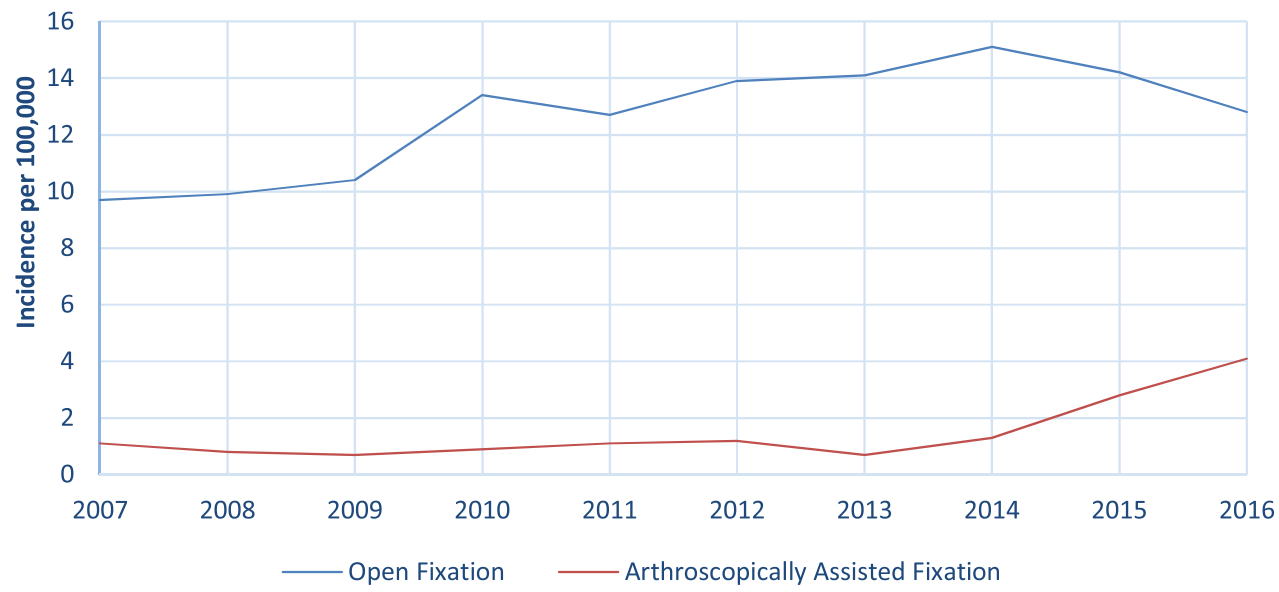

\section{Patient Demographics}

After propensity matching, 952 patients were included in the study for further analysis. There were 476 patients in the AATPFF cohort and 476 patients in the traditional fixation cohort. As a result of the propensity matching, all examined patient demographics and comorbidities did not differ between the AATPFF and traditional tibial plateau fixation cohorts (Table 1).

\section{Complications}

There was a significantly greater incidence of minor medical complications $(8.2 \%$ vs $2.7 \%, P=.0002)$, major medical complications $(9.9 \%$ vs $4.6 \%, P=.0018)$, surgical complications $(13.2 \%$ vs $2.7 \%, P<.0001)$, and emergency department visits $(21.4 \%$ vs $13.5 \%, P=.0012)$ within 90 days of surgery in the traditional tibial plateau fixation cohort compared with AATPFF (Table 2). However, there was no difference in reoperation rates between the 2 cohorts $(2.9 \%$ vs $3.6 \%, P=.8504)$.

\section{Logistic Regression Model}

A multivariate logistic regression analysis was performed to determine independent risk factors associated with reoperation rate within 90 days after index tibial plateau fixation. In this multivariable model, diabetes mellitus (odds ratio 6.4; 95\% confidence interval 1.21-45.1; $P=.0409$ ) was the only risk factor associated with reoperation within 90 days after index surgery. Age, sex, and other preoperative comorbidities were not associated with increased reoperation rates after surgery.

\section{Discussion}

Between 2006 and 2017, the use of AATPFF methods increased at a significantly faster rate than the traditional method of open reduction and internal fixation. Regardless of treatment strategy, the incidence of tibial plateau fracture fixation increased over the study period. Furthermore, the addition of knee arthroscopy to tibial plateau open reduction and internal fixation did not increase the risk of medical complications, surgical complications, postoperative emergency department visits, or reoperation.

The etiology of the increased fixation of tibial plateau fractures is likely multifactorial; however, we speculate that 2 explanations remain likely. First, there has been an increase in the incidence of tibial plateau fractures over time. Tibial plateau fractures account for approximately $1 \%$ of all fractures and $8 \%$ of fractures in the elderly population. ${ }^{1}$ It has been well described that older patients with osteoporotic bone are at increased risk of tibial plateau injury with low-energy mechanisms. ${ }^{1-3}$ With contemporary improvements in health care delivery and access in the setting of the "baby boomer" generation, more people are living longer than ever before leading to an ever-increasing population prone to these fractures. ${ }^{17}$ Second, there has been a movement away from nonoperative management of tibial plateau fractures. With the advances in orthopaedic implants, minimally invasive procedures, and soft-tissue handling techniques, there has been a decrease in complication rates compared with older studies using large approaches in compromised soft tissues. ${ }^{18}$

While the incidence of tibial plateau fracture fixation is increasing, the use of knee arthroscopy as a reduction tool is increasing at a faster rate compared with traditional techniques. Unlike sports medicine-trained orthopaedic surgeons, knee arthroscopy is not a technique frequently used by orthopaedic traumatologists. ${ }^{19}$ Examining the annual trends in AATPFF (Fig 1) reveals a marked 
Table 1. Comparison of Patient Demographics Between Arthroscopically Assisted and Traditional Tibial Plateau Fixation Cohorts

\begin{tabular}{|c|c|c|c|}
\hline Patient Characteristics & $\begin{array}{l}\text { Arthroscopically Assisted Tibial } \\
\text { Plateau Fracture Fixation, } \mathrm{n}=476(50 \%)\end{array}$ & $\begin{array}{l}\text { Traditional Tibial } \\
\text { Fracture Fixation, } \mathrm{n}=476(50 \%) \\
\end{array}$ & $P$ Value \\
\hline Age, $>65$ y & $241(50.6)$ & $241(50.6)$ & 1.000 \\
\hline Male sex, n (\%) & $196(41.2)$ & $196(41.2)$ & 1.000 \\
\hline Obese, n (\%) & $131(27.5)$ & $111(23.3)$ & .1366 \\
\hline Diabetes mellitus, n (\%) & $119(25)$ & $110(23.1)$ & .495 \\
\hline Hypertension, n (\%) & $296(62.2)$ & $282(59.2)$ & .3529 \\
\hline Congestive heart failure, n (\%) & $27(5.7)$ & $34(7.1)$ & .3542 \\
\hline Prior myocardial infarction (\%) & $12(2.5)$ & $15(3.2)$ & .5581 \\
\hline Chronic obstructive pulmonary disease, $\mathrm{n}(\%)$ & $25(5.3)$ & $26(5.5)$ & .8856 \\
\hline Peripheral vascular disease, n (\%) & $59(12.4)$ & $63(13.2)$ & .6981 \\
\hline Preoperative sepsis, n (\%) & $11(2.3)$ & $19(4)$ & .1378 \\
\hline Fibromyalgia, n (\%) & $56(11.8)$ & $38(8)$ & .051 \\
\hline Depression or anxiety, n (\%) & $141(29.6)$ & $116(24.4)$ & .068 \\
\hline Smoking history, $\mathrm{n}(\%)$ & $66(13.9)$ & $55(11.6)$ & .2845 \\
\hline
\end{tabular}

increase in the use of the arthroscopic-assisted technique after the year 2013. At that time, the Accreditation Council for Graduate Medical Education implemented case minimum benchmarks for select procedures that must be met by all orthopaedic surgery residents before graduation from an accredited 5-year residency program. ${ }^{20,21}$ Knee arthroscopy was among the procedures selected by the Accreditation Council for Graduate Medical Education with at least 30 cases required before graduation. This mandated exposure to knee arthroscopy may have contributed to the increased use of AATPFF as residency graduates after 2013 would have been motivated to gain experience and familiarity with the arthroscopic technique.

Our study failed to demonstrate an increased risk of postoperative medical complications with AATPFF compared with traditional open reduction and internal fixation. In fact, the traditional fixation group demonstrated greater rates of postoperative complications compared with the AATPFF cohort, which is almost certainly secondary to our inability to control for fracture pattern, severity, or associated soft-tissue injury. In a study of 519 tibial plateau fractures, Basques et al. ${ }^{22}$ reported a $10.2 \%$ total medical complication rate and a $7.3 \%$ rate of major medical complications, which is consistent with the findings in our traditional fixation cohort. Using bivariate analysis, the occurrence of medical complications following tibial plateau fixation in the previously mentioned study was associated with bicondylar fractures (Schatzker V-VI). ${ }^{22}$ AATPFF has been widely accepted as a safe and effective treatment option for unicondylar injuries (Schatzker I-IV), with most of the supporting literature examining lateral based fracture patterns (Schatzker I-III). ${ }^{10-16,23,24}$ One author stated that Schatzker V-VI injuries represented contraindications to AATPFF and instead knee arthroscopy should only be performed on lateral column injuries. ${ }^{25}$ Similarly, Herbort et al. reported that these high-energy complex fracture patterns are contraindications to knee arthroscopy given case reports of iatrogenic compartment syndrome from arthroscopy fluid extravasation into the lower leg. ${ }^{23,26,27}$

Similar to postoperative medical complications, the addition of knee arthroscopy to tibial plateau fracture fixation did not increase the risk of surgical complications. High-energy injury mechanisms often lead to a traumatized and compromised soft-tissue envelope, which places these patients at a high risk of infection following surgery. Bachoura et al. ${ }^{28}$ examined 1611 patients who underwent 1783 surgeries related to orthopaedic trauma over a 3-year study period and found that tibial plateau fractures had an incidence of surgical site infections more than 2 -fold greater than any other orthopaedic injury. Furthermore, Basques et al. ${ }^{22}$ reported that bicondylar fracture patterns independently predict postoperative infection. Similar to our findings,

Table 2. Comparison of Complication Rates Within 90 Days Between Arthroscopically Assisted and Traditional Tibial Plateau Fixation Cohorts

\begin{tabular}{|c|c|c|c|}
\hline Complications & $\begin{array}{l}\text { Arthroscopically Assisted Tibial } \\
\text { Plateau Fracture Fixation, } n=476(\%)\end{array}$ & $\begin{array}{l}\text { Traditional Tibial } \\
\text { Fracture Fixation, } \mathrm{n}=476(\%)\end{array}$ & $P$ Value \\
\hline$\overline{\text { Return to OR, n (\%) }}$ & $15(3.2)$ & $14(2.9)$ & .8504 \\
\hline Minor medical complications, n (\%) & $13(2.7)$ & $39(8.2)$ & .0002 \\
\hline Surgical complications, n (\%) & $13(2.7)$ & $63(13.2)$ & $<.0001^{*}$ \\
\hline Emergency department visits, n (\%) & $64(13.4)$ & $102(21.4)$ & .0012 \\
\hline
\end{tabular}

OR, operating room.

*Statistically significant. 
Dall'oca et al. ${ }^{12}$ reported a lower incidence of infection in bicondylar injuries with AATPFF compared with traditional open reduction and internal fixation in a series of 100 tibial plateau fractures. Contrary to our study, Elabjer et al. ${ }^{29}$ examined 78 patients with lateral unicondylar fractures (Schatzker I-III) and found no difference in rates of postoperative infection. Regardless, there have been no reports that suggest that the addition of knee arthroscopy to tibial plateau fracture fixation increases the rate of infection.

In addition, we found no difference in rates of reoperation within 90 days between AATPFF and traditional methods. On multivariate analysis, diabetes was the only identified risk factor for reoperation. Age, sex, and other preoperative comorbidities were not associated with increased reoperation rates after tibial plateau fracture fixation. Diabetes was previously identified in another large database study of tibial plateau fractures to be a risk factor for readmission, however reoperation was not examined in the mentioned study. ${ }^{22}$ Henry et al. ${ }^{30}$ retrospectively reviewed a cohort of 8426 patients with tibial plateau fractures who underwent unilateral open reduction and internal fixation. In their study, the need for revision fixation was increased significantly by the presence of open fracture, bicondylar fracture pattern, concomitant tibial shaft fracture, and surgery occurring after midnight or during the evening/weekend. ${ }^{30}$ Subsequent irrigation and debridement was predicted by open injury, bicondylar fracture pattern, temporizing external fixator, and concomitant tibial-shaft fracture. ${ }^{30}$ Interestingly, the development of compartment syndrome was not associated with infection in their large cohort, which is in contrast to other studies. ${ }^{30-33}$ Unfortunately, we were unable to assess similar variables using the PearlDiver software.

\section{Limitations}

Limitations of the current study include those inherent to its retrospective study design and use of a large administrative claims database. Such studies rely on accurate billing and coding as well as correctly reported patient demographic information. Furthermore, detailed radiographic, clinical, and fracture specific factors such as open injury, soft-tissue injury, fracture pattern, degree of articular impaction or comminution, fracture displacement, and polytrauma status could not be assessed. Additional operative variables such as operative time, surgical approach, quality of fixation, and quality of reduction could not be evaluated. Lastly, patient follow-up including treatment of postoperative complications that occurred outside of the institution participating in database data collection would be missed based on study design.

\section{Conclusions}

The incidence of tibial plateau fracture fixation is increasing; however, the use of AATPFF is increasing at a faster rate compared with traditional techniques. Furthermore, the addition of knee arthroscopy to fracture fixation does not increase the risk of complication, reoperation, or emergency department visit within 90 days.

\section{References}

1. Court-Brown CM, Caesar B. Epidemiology of adult fractures: A review. Injury 2006;37:691-697.

2. He QF, Sun H, Shu LY, et al. Tibial plateau fractures in elderly people: an institutional retrospective study. J Orthop Surg Res 2018;13:276.

3. Marongiu G, Mastio M, Capone A. Current options to surgical treatment in osteoporotic fractures. Aging Clin Exp Res 2013;25:S15-S17 (suppl 1).

4. Lubowitz JH, Elson WS, Guttmann D. Part I: Arthroscopic management of tibial plateau fractures. Arthroscopy 2004;20:1063-1070.

5. Krause M, Preiss A, Meenen NM, Madert J, Frosch KH. "Fracturoscopy" is superior to fluoroscopy in the articular reconstruction of complex tibial plateau fractures-an arthroscopy assisted fracture reduction technique. J Orthop Trauma 2016;30:437-444.

6. Kokkalis ZT, Iliopoulos ID, Pantazis C, Panagiotopoulos E. What's new in the management of complex tibial plateau fractures? Injury 2016;47:1162-1169.

7. Jennings JE. Arthroscopic management of tibial plateau fractures. Arthroscopy 1985;1:160-168.

8. Caspari RB, Hutton PM, Whipple TL, Meyers JF. The role of arthroscopy in the management of tibial plateau fractures. Arthroscopy 1985;1:76-82.

9. Chase R, Usmani K, Shahi A, Graf K, Mashru R. Arthroscopic-assisted reduction of tibial plateau fractures. Orthop Clin North Am 2019;50:305-314.

10. Chan YS, Yuan LJ, Hung SS, et al. Arthroscopic-assisted reduction with bilateral buttress plate fixation of complex tibial plateau fractures. Arthroscopy 2003;19:974-984.

11. Chiu CH, Cheng CY, Tsai MC, et al. Arthroscopy-assisted reduction of posteromedial tibial plateau fractures with buttress plate and cannulated screw construct. Arthroscopy 2013;29:1346-1354.

12. Dall'oca C, Maluta T, Lavini F, Bondi M, Micheloni GM, Bartolozzi P. Tibial plateau fractures: Compared outcomes between ARIF and ORIF. Strategies Trauma Limb Reconstr 2012;7:163-175.

13. Duan XJ, Yang L, Guo L, Chen GX, Dai G. Arthroscopically assisted treatment for Schatzker type I-V tibial plateau fractures. Chin J Traumatol 2008;11:288-292.

14. Kayali C, Ozturk H, Altay T, Reisoglu A, Agus H. Arthroscopically assisted percutaneous osteosynthesis of lateral tibial plateau fractures. Can J Surg 2008;51: 378-382.

15. Levy BA, Herrera DA, Macdonald P, Cole PA. The medial approach for arthroscopic-assisted fixation of lateral tibial plateau fractures: Patient selection and mid- to long-term results. J Orthop Trauma 2008;22:201-205.

16. Ruiz-Iban MA, Diaz-Heredia J, Elias-Martin E, MorosMarco S, Cebreiro Martinez Del Val I. Repair of meniscal tears associated with tibial plateau fractures: A review of 15 cases. Am J Sports Med 2012;40:2289-2295. 
17. Crimmins EM, Zhang Y, Saito Y. Trends over 4 decades in disability-free life expectancy in the United States. Am J Public Health 2016;106:1287-1293.

18. Prat-Fabregat S, Camacho-Carrasco P. Treatment strategy for tibial plateau fractures: An update. EFORT Open Rev 2016;1:225-232.

19. Degen RM, Lebedeva Y, Birmingham TB, et al. Trends in knee arthroscopy utilization: A gap in knowledge translation. Knee Surg Sports Traumatol Arthrosc 2020;28:439-447.

20. Carender CN, Shamrock AG, An Q, Karam MD. Variability in experience performing lower extremity amputations between surgical residents: An examination of ACGME case logs. Iowa Orthop J 2019;39:15-20.

21. Hinds RM, Gottschalk MB, Strauss EJ, Capo JT. Trends in arthroscopic procedures performed during orthopaedic residency: An analysis of Accreditation Council for Graduate Medical Education case log data. Arthroscopy 2016;32:645-650.

22. Basques BA, Webb ML, Bohl DD, Golinvaux NS, Grauer JN. Adverse events, length of stay, and readmission after surgery for tibial plateau fractures. J Orthop Trauma 2015;29:e121-e126.

23. Herbort M, Domnick C, Petersen W. Arthroscopic treatment of tibial plateau fractures. Oper Orthop Traumatol 2014;26:573-588. quiz 589-590 [in German].

24. Burdin G. Arthroscopic management of tibial plateau fractures: Surgical technique. Orthop Traumatol Surg Res 2013;99:S208-S218.

25. Tornetta P 3rd. Arthroscopic elevation with grafting. J Orthop Trauma 2002;16:444-446.
26. Belanger M, Fadale P. Compartment syndrome of the leg after arthroscopic examination of a tibial plateau fracture. Case report and review of the literature. Arthroscopy 1997;13:646-651.

27. Kiefer H, Zivaljevic N, Imbriglia JE. Arthroscopic reduction and internal fixation (ARIF) of lateral tibial plateau fractures. Knee Surg Sports Traumatol Arthrosc 2001;9:167-172.

28. Bachoura A, Guitton TG, Smith RM, Vrahas MS, Zurakowski D, Ring D. Infirmity and injury complexity are risk factors for surgical-site infection after operative fracture care. Clin Orthop Rel Res 201 1;469:2621-2630.

29. Elabjer E, Bencic I, Cuti T, Cerovecki T, Curic S, Vidovic D. Tibial plateau fracture management: Arthroscopicallyassisted versus ORIF procedure-clinical and radiological comparison. Injury 2017;48:S61-S64 (suppl 5).

30. Henry P, Wasserstein D, Paterson M, Kreder H, Jenkinson R. Risk factors for reoperation and mortality after the operative treatment of tibial plateau fractures in Ontario, 1996-2009. J Orthop Trauma 2015;29:182-188.

31. Shao J, Chang H, Zhu Y, et al. Incidence and risk factors for surgical site infection after open reduction and internal fixation of tibial plateau fracture: A systematic review and meta-analysis. Int J Surg 2017;41:176-182.

32. Lin S, Mauffrey C, Hammerberg EM, Stahel PF, Hak DJ. Surgical site infection after open reduction and internal fixation of tibial plateau fractures. Eur J Orthop Surg Traumatol 2014;24:797-803.

33. Momaya AM, Hlavacek J, Etier B, et al. Risk factors for infection after operative fixation of tbial plateau fractures. Injury 2016:47:1501-1505. 